\title{
Wo ist das Land der Aufstiegschancen?
}

\section{Margaret Davenport}

\section{Relevanz}

Ungleichheit zu verringern ist eine zentrale Aufgabe der Politik. Mit progressiven Steuern oben und einem Einkommenszuschuss unten schaffen wir den Ausgleich. Noch besser ist es, Ungleichheit durch Armut möglichst wenig entstehen zu lassen. Ungleichheit ist keine statische Angelegenheit und Armut kein Schicksal. Sozialer Aufstieg und Abstieg verändern die Position in der Gesellschaft. Wer heute arm ist und seine Chance nutzen kann, bringt es morgen zu Wohlstand. Talent und Umgebung sind wichtig, ein stabiles Elternhaus, leistungsfähige Schulen, eine inspirierende Umgebung mit vielen Erfolgsgeschichten, und eine Politik, die den Aufstieg fördert. Dazu gehört eine Kultur des fairen Wettbewerbs, die den Zugang zu Bildung und lukrativen Berufen ebnet, den Weg in die Selbstständigkeit erleichtert, Diskriminierung und unlauteren Wettbewerb bekämpft, und die Umverteilungspolitik mit Leistungsanreizen verbindet. Das wäre ein Land der Aufsteiger.

Christian Keuschnigg

\section{Quelle}

Der nachfolgende Text ist eine Zusammenfassung von: Chetty, Raj, Nathaniel Hendren, Patrick Kline und Emmanuel Saez (2014), Where is the Land of

M. Davenport $(\bowtie)$

Universität St. Gallen, St. Gallen, Schweiz

E-Mail: margaret.davenport@unisg.ch 
Opportunity? The Geography of Intergenerational Mobility in the United States, Quarterly Journal of Economics 129, 1553-1623.

„Behaltet, o alte Lande, euren sagenumwobenen Prunk“, ruft sie mit stummen Lippen. „Gebt mir eure müden, eure armen, eure geknechteten Massen, die frei zu atmen begehren, den elenden Unrat eurer gedrängten Küsten; Schickt sie mir, die Heimatlosen, vom Sturme Getriebenen, hoch halt' ich mein Licht am goldenen Tore!“ (Emma Lazarus, The New Colossus).

Dieses Zitat der amerikanische Dichterin Emma Lazarus steht am Fuße der Freiheitsstatue im Hafen von New York und dient noch heute als Sinnbild für all jene, die den „American Dream“ suchen. Einmal im Land, so glaubten und glauben noch viele, wartet unabhängig von bescheidenen Anfängen eine Welt von Chancen und ein Versprechen von Freiheit.

Das Streben einer Gesellschaft nach Freiheit ist auch eine Voraussetzung für den Einzelnen, erfolgreich zu sein. Der Erfolg der nächsten Generation soll nicht vom Einkommen und vom sozialen Status der Eltern abhängen. Den Kindern aus allen Einkommensschichten sollen die gleichen sozialen und wirtschaftlichen Aufstiegsmöglichkeiten offenstehen. Ein hohes Maß an Aufstiegsmöglichkeiten von Generation zu Generation ist ein Symbol für Chancengleichheit und soziale Gerechtigkeit. Obwohl heute diese Chancengleichheit vielfach nur mehr anekdotenhaft belegt ist, glauben viele Amerikaner immer noch, dass dem Einzelnen alle Möglichkeiten offenstehen.

Trotz dieser anhaltenden Überzeugung widerlegt die Studie, dass die amerikanische Gesellschaft einen überdurchschnittlichen Grad an generationenübergreifenden Aufstiegschancen aufweist. Die Autoren zeigen, dass die Vereinigten Staaten geringere Aufstiegsmöglichkeiten zwischen Generationen aufweisen als vielfach angenommen. So liegt die Wahrscheinlichkeit bei etwa 7,5\%, dass Kinder aus einer Familie aus den untersten $20 \%$ der Einkommensverteilung im erwachsenen Alter in die Top $20 \%$ der Verteilung gelangen. Dagegen liegt diese Aufstiegswahrscheinlichkeit in Kanada bei 13,4 \% und in Dänemark bei 11,4\%. Außerdem weist die durchschnittliche Aufstiegswahrscheinlichkeit erhebliche geographische Unterschiede innerhalb der U.S.A. auf. In manchen Städten sind die Aufstiegschancen sehr hoch, vielfach höher als in europäischen Städten vergleichbarer Größe. Anderswo sind die Aufstiegschancen wiederum so gering, dass dort nur wenige der Armut je entfliehen werden.

Die Wahrscheinlichkeit, dass Kinder aus einer Familie der $20 \%$ tiefsten Einkommen im Laufe des Lebens in die Top $20 \%$ aufsteigen, beträgt in den U.S.A. etwa 7,5\%. Dagegen liegen diese Aufstiegschancen in Kanada bei 13,4 und in Dänemark bei 11,4\%. 
Die Autoren nutzen Informationen über 40 Mio. Kinder und den dazugehörenden elterlichen Steuerdaten für den Zeitraum 1996 bis 2012. Dabei wird das Einkommen der Eltern, wenn ihre Kinder zwischen 15 und 20 Jahre alt sind, mit demjenigen der Kinder verglichen, wenn diese das 30. Lebensjahr erreichen. Jedes Kind im Alter von 16 Jahren wird einem geographischen Gebiet bzw. einer Pendlerzone zugeordnet. Dafür werden einzig jene Kinder berücksichtigt, welche aus einem Haushalt stammen, in dem das elterliche Einkommen im untersten Viertel der Einkommensverteilung liegt, also weniger als \$28,800 beträgt. Dann wird eine Rangordnung der Pendlerzonen nach dem Grad der dort vorherrschenden sozialen Mobilität gebildet. So kann ermittelt werden, inwiefern das zukünftige Einkommensniveau vom Wohnort als Kind abhängig ist. Es zeigen sich erhebliche Unterschiede nach Regionen: Kinder, welche in Städten mit einer hohen Mobilität aufgewachsen sind, wie z. B. Salt Lake City, erreichen ab ihrem 30. bis 32. Lebensjahr ein Einkommen, welches in der Einkommensverteilung um 10 Prozentpunkte höher liegt als das Einkommen von Kindern aus Regionen mit tiefer Mobilität. So können Kinder, welche in den obersten $10 \%$ der Pendlerzonen mit der höchsten sozialen Mobilität aufwachsen, mit 30 Jahren ein Jahreseinkommen von mehr als \$ 35.000 erwarten. Dagegen können Kinder aus den untersten $10 \%$ der Gebiete mit der niedrigsten sozialen Mobilität nicht mehr als \$22.900 erreichen.

Kinder, die in Städten mit hoher Mobilität aufwachsen, erzielen ab ihrem 30. bis 32. Lebensjahr ein Einkommen, welches in der Einkommensverteilung um 10 Prozentpunkte höher liegt als die Einkommen von Kindern aus Regionen mit geringer Mobilität.

Welche regionalen Unterschiede bestehen innerhalb der Vereinigten Staaten konkret? Die Aufstiegschancen sind im Präriegebiet des Westens am höchsten und im Südosten des Landes am niedrigsten. Die Westküste und der Nordosten weisen ebenfalls einen hohen Grad an sozialer Mobilität auf. Betrachtet man die 50 größten Pendlerzonen, dann sind die Gegensätze zwischen Salt Lake City und Charlotte am größten, wobei diese beiden Städte an entgegengesetzten Enden des Kontinents liegen. Die geographische Distanz ist allerdings nicht maßgebend für das Ergebnis: so liegt zum Beispiel Pittsburgh in Pennsylvania auf Rang 2 und Cleveland in Ohio auf Rang 40, zwei Städte, die nur 216 km oder $73 \%$ der Fahrdistanz zwischen Salzburg und Wien auseinanderliegen. Im Durchschnitt weisen städtische Gebiete ein tieferes Maß an Aufstiegsmöglichkeiten zwischen Generationen auf als ländliche Gebiete. 
Die Ergebnisse hängen stark von Faktoren ab, welche bereits vor dem Eintritt in den Arbeitsmarkt weitgehend festgezurrt sind. Der soziale Aufstieg korreliert stark mit Faktoren wie Hochschulbesuchsquoten, Qualität der besuchten Hochschule oder dem Anteil jugendlicher Eltern, die noch über ein langes Arbeitsleben Einkommen verdienen können. Von allen Kindern mit 30 Jahren leben $38 \%$ an einem anderen Ort als dort, wo sie aufgewachsen sind. Trotz dieser hohen geographischen Mobilität sind die Unterschiede im Einkommen der Kinder weiterhin abhängig von lange nachwirkenden Faktoren, welche bereits vorhanden sind, bevor ein Kind das Elternhaus verlässt, eine Hochschule besucht oder in den Arbeitsmarkt eintritt.

Die Frage ist, welche weiteren Faktoren die sozialen Aufstiegsmöglichkeiten besonders stark beeinflussen. Die Studie identifiziert fünf Faktoren, welche die Aufstiegschancen mitbestimmen: Segregation, Ungleichheit, Schulqualität, Sozialkapital und Familienstruktur. Diese erklären 76 \% der Unterschiede in der sozialen Mobilität zwischen den einzelnen Pendlerzonen.

Segregation, Ungleichheit, Schulqualität, Sozialkapital und Familienstruktur können $76 \%$ der Unterschiede in den sozialen Aufstiegschancen zwischen verschiedenen Regionen erklären.

Gebiete in den Vereinigten Staaten mit geringen Aufstiegsmöglichkeiten werden jeweils nach den zwei Dimensionen Rasse und Einkommen unterteilt. In Gebieten mit einem hohen Anteil an Afroamerikanern haben sowohl die schwarze als auch die weiße Bevölkerung niedrige Aufstiegschancen, beeinflusst durch die vielfach mäßige Qualität der dortigen sozialen Einrichtungen. Eine geographische Trennung der Armen von der Mittelklasse und den Reichen hat einen großen negativen Einfluss auf die sozialen Aufstiegschancen des Einzelnen. Ein mögliches Maß für diese Segregation ist die benötigte Zeit für den Weg zwischen Wohn- und Arbeitsort. Bewohner von Gebieten mit geringen Aufstiegschancen haben typischerweise lange Arbeitswege und sie verfügen vielfach über nur einfache öffentliche Verkehrsinfrastrukturen.

Regionen mit weniger Aufstiegschancen haben oft eine kleine Mittelschicht und weisen einen höheren Grad an Einkommensungleichheit auf. Die Studie stellt fest, dass die Größe des Mittelstands, gemessen an der Differenz zwischen dem obersten und untersten Viertel der Einkommensverteilung, besonders wichtig ist und nicht etwa die vielfach genannten, einkommensstärksten $1 \%$ der Bevölkerung. Nicht die Einkommensungleichheit an sich verringert die Aufstiegschancen, sondern vor allem diejenigen Faktoren, welche den Mittelstand aushöhlen. 
Bessere schulische Leistungen und geringe Schulabbruchsquoten fördern den sozialen Aufstieg. Typischerweise sind die Aufstiegschancen höher in Pendlerzonen mit hoher Mobilität, in Gebieten mit hohen Wahlbeteiligungen und in Gemeinden mit einem hohen religiösen Bewusstsein. Schließlich spielen auch stabile Familienverhältnisse eine zentrale Rolle in der Entwicklung der Kinder, insbesondere bevor diese in die Arbeitswelt eintreten. Hält man alle anderen Faktoren konstant, dann haben Kinder eines alleinerziehenden Elternteils geringere Aufstiegschancen. Das gilt auch für die gesamte Gemeinschaft. Ein höherer Anteil an Kindern, welche von einem alleinerziehenden Elternteil aufgezogen werden, beeinflusst die Aufstiegschancen in der Region negativ.

Open Access Dieses Kapitel wird unter der Creative Commons Namensnennung 4.0 International Lizenz (http://creativecommons.org/licenses/by/4.0/deed.de) veröffentlicht, welche die Nutzung, Vervielfältigung, Bearbeitung, Verbreitung und Wiedergabe in jeglichem Medium und Format erlaubt, sofern Sie den/die ursprünglichen Autor(en) und die Quelle ordnungsgemäß nennen, einen Link zur Creative Commons Lizenz beifügen und angeben, ob Änderungen vorgenommen wurden.

Die in diesem Kapitel enthaltenen Bilder und sonstiges Drittmaterial unterliegen ebenfalls der genannten Creative Commons Lizenz, sofern sich aus der Abbildungslegende nichts anderes ergibt. Sofern das betreffende Material nicht unter der genannten Creative Commons Lizenz steht und die betreffende Handlung nicht nach gesetzlichen Vorschriften erlaubt ist, ist für die oben aufgeführten Weiterverwendungen des Materials die Einwilligung des jeweiligen Rechteinhabers einzuholen.

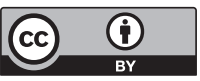

